\title{
Primary Rat Hepatocyte Culture on 3D Nanofibrous Polymer Scaffolds for Toxicology and Pharmaceutical Research
}

\author{
Jeanette Bierwolf, ${ }^{1}$ Marc Lutgehetmann, ${ }^{2}$ Kai Feng, ${ }^{3}$ Johannes Erbes, ${ }^{1}$ \\ Steffen Deichmann, ${ }^{1}$ Eva Toronyi, ${ }^{1}$ Christina Stieglitz, ${ }^{1}$ Bjoern Nashan, ${ }^{1}$ Peter X. Ma, ${ }^{4}$ \\ Joerg M. Pollok ${ }^{1}$ \\ ${ }^{1}$ Department of Hepatobiliary and Transplant Surgery, University Medical Center Hamburg- \\ Eppendorf, Martinistrasse 52, 20246 Hamburg, Germany; telephone: +49-40-7410-58572; \\ fax: +49-40-7410-45311; e-mail: pollok@uke.de \\ ${ }^{2}$ Department of Internal Medicine and Department of Medical Microbiology, Virology and \\ Hygiene, University Medical Center Hamburg-Eppendorf, Hamburg, Germany \\ ${ }^{3}$ University of Michigan, Macromolecular Science and Engineering Center, Ann Arbor, \\ Michigan \\ ${ }^{4}$ Department of Biologic and Materials Sciences, Department of Biomedical Engineering, \\ University of Michigan, Macromolecular Science and Engineering Center, 1011 North \\ University Ave, Room 2211, Ann Arbor, Michigan 48109-1078; telephone: +1-734-764-2209; \\ fax: +1-734-647-2110; e-mail: mapx@umich.edu
}

Received 4 June 2010; revision received 29 July 2010; accepted 26 August 2010

Published online 7 September 2010 in Wiley Online Library (wileyonlinelibrary.com). DOI 10.1002/bit.22924

ABSTRACT: Primary rat hepatocytes are a widely used experimental model to estimate drug metabolism and toxicity. In currently used two-dimensional (2D) cell culture systems, typical problems like morphological changes and the loss of liver cell-specific functions occur. We hypothesize that the use of polymer scaffolds could overcome these problems and support the establishment of three-dimensional (3D) culture systems in pharmaceutical research. Isolated primary rat hepatocytes were cultured on collagen-coated nanofibrous scaffolds for 7 days. Cell loading efficiency was quantified via DNA content measurement. Cell viability and presence of liver-cell-specific functions (albumin secretion, glycogen storage capacity) were evaluated. The activity of liver-specific factors was analyzed by immunofluorescent staining. RNA was isolated to establish quantitative real-time PCR. Our results indicate that primary rat hepatocytes cultured on nanofibrous scaffolds revealed high viability and well-preserved glycogen storage. Albumin secretion was existent during the entire culture period. Hepatocytes remain HNF-4 positive, indicating highly preserved cell differentiation. Aggregated hepatocytes re-established positive signaling for Connexin 32, a marker for differentiated hepatocyte interaction. ZO-1-positive hepatocytes were detected indicating formation of tight junctions. Expression of cytochrome isoenzymes was inducible. Altogether the data suggest that nanofibrous scaffolds

Correspondence to: Joerg M. Pollok or Peter X. Ma

Contract grant sponsor: National Institutes of Health (NIH)

Contract grant number: NIGMS GM075840 provide a good in vitro microenvironment for neo tissue regeneration of primary rat hepatocytes.

Biotechnol. Bioeng. 2011;108: 141-150.

(C) 2010 Wiley Periodicals, Inc.

KEYWORDS: tissue engineering; hepatocytes; nano; polymer scaffolds; pharmacology; toxicology

\section{Introduction}

Understanding of drug metabolism and transport is critically important for the evaluation of safety and efficacy of new drug candidates. Although animal experiments and human clinical trials are necessary, these studies are expensive and ethically controversial. In vitro investigation of the metabolic profile of a drug is more economical, easier to carry out, and less controversial. Therefore, toxicology studies using isolated hepatocytes are recognized as one of the most relevant and practical models (Hewitt et al., 2007). Freshly isolated primary rat hepatocytes are a widely used experimental equipment to experimentally estimate drug metabolism and toxicity due to their high metabolic function (Aly and Domenech, 2009; Ellis et al., 2010; Li et al., 2009; Santos et al., 2009; Shitara et al., 2009). In currently used two-dimensional (2D) culture systems 
typical problems such as low seeding efficiency, morphological changes (Hamilton et al., 2001; Hewitt et al., 2007), and the loss of liver-cell-specific functions occur (LeCluyse et al., 2005). In our previous studies we successfully cultured primary rat hepatocytes on three-dimensional (3D) biodegradable polymer scaffolds (Pollok, 2003; Pollok et al., 1998; Torok et al., 2001a,b, 2002, 2006). Various scaffold fabrication techniques have been developed as reviewed elsewhere (Liu et al., 2006; Ma, 2003, 2004). The high cell seeding efficiency and the maintenance of differentiated function of the incorporated cells are important criteria for scaffolds. A novel preparation technique to create 3D nanofibrous poly-(L-lactic acid) (PLLA) scaffolds with wellcontrolled interconnected spherical micropores has been developed by $\mathrm{Ma}$ et al. (Chen and Ma, 2004; Wei and Ma, 2006). This new processing technique, initially evaluated using osteoblastic progenitor cells, enables a unique combination of a high porosity $(96 \%)$, spherical pore shape, controlled pore size, interpore connectivity, and nanofibrous pore wall architecture. The nanofibrous scaffold architecture increased cell attachment and enhanced osteoblastic marker gene expression, indicating increased differentiation of the osteoblastic cells compared to scaffolds without the nanofibrous feature as described previously (Chen et al., 2006; Lee et al., 2009; Ma, 2008; Woo et al., 2007).

The nano scaffold attempts to mimic the fine fibrous architecture of collagen, a natural extracellular matrix component of nearly every tissue. The fibrillar structure of collagen may be important for cell attachment, proliferation, and differentiated function in culture (Smith and Ma, 2004). Contrary to scaffolds, produced by mixing collagen I and III from bovine hide (as fabricated by BD Bioscience, Franklin Lakes, NJ), the nano scaffolds used in the presented experiments are free of animal material, avoiding interactions between different species. Other scaffold fabrication techniques, as solid-walled scaffolds have been described; however, cells attached on nanofibrous scaffolds $70 \%$ higher than on solid-walled scaffolds (Woo et al., 2003). Nanofibrous scaffolds have a high surface-to-volume ratio which is thought to enhance cell adhesion. There is a wide range of 3D liver cell culture models used in the attempt to create an in vivo like environment for the culture hepatocytes (primary hepatocyte, immortalized hepatocyte cell lines, stem cell derived hepatocyte-like cells). The cells are cultured under static culture conditions or in perfusion systems. As scaffolds either natural materials like alginate, or collagen, or synthetic materials like PLLA, poly(glycolic acid), poly( $\varepsilon$-caprolactone), or poly(hydroxy butyrate) (Ma, 2004) are used with their respective advantages and disadvantages, such as pore size, mechanical handling, interspecies interaction, or definition of composition. Starting from the favorable experience with osteoblast culture on the nanofibrous scaffolds, developed by Ma et al. (Chen and Ma, 2004; Wei and Ma, 2006), we anticipated that this matrix will also advantageously support hepatocyte adhesion, differentiated function, and formation of neo liver tissue.
In addition to the extracellular matrix application, an initially high cell density has a wide influence on morphology and in vivo like function of the resultant neo liver tissue. On the other hand, the pharmaceutical industry needs effective and robust assays with high-throughput testing to speed up the evaluation of new drugs, requiring utilization of 96-well formats (Gebhardt et al., 2003; Hertzberg and Pope, 2000; Hewitt et al., 2007). The restricted growth area in one well of a 96-well microplate forecloses high initially cell seeding. Highdensity seeding of cells on polymer scaffolds can be achieved by centrifugal force seeding method (Dvir-Ginzberg et al., 2003; Yang et al., 2001). In this context we reason that tissue engineering of primary rat hepatocytes using nanofibrous scaffolds is superior to monolayer culture and could support the establishment of 3D culture systems in pharmaceutical research due to morphological and functional stability.

\section{Materials and Methods}

\section{Scaffold Fabrication}

Nanofibrous PLLA scaffolds with interconnected pores were produced using a phase separation technique as described previously (Chen and Ma, 2004; Ma and Choi, 2001; Ma and Zhang, 1999; Wei and Ma, 2006). Scaffolds were created to fit in 96-well microplates $(6 \mathrm{~mm}$ in diameter and $2.0 \mathrm{~mm}$ in thickness). To increase hydrophilicity, the scaffolds were treated as follows: $80 \%$ ethanol (Geyer, Renningen, Germany) under careful vacuum treatment until the scaffolds sank to the bottom. Fifty percent ethanol $/ 50 \%$ PBS buffer (Invitrogen, Carlsbad, CA) in the same manner, followed by $100 \%$ PBS buffer for $2 \mathrm{~min}$. After this procedure, the scaffolds were transferred into a Petri dish and coated with a buffer/collagen (Serva, Heidelberg, Germany) solution (ratio 10:1) for $10 \mathrm{~min}$.

\section{Isolation of Rat Hepatocytes}

The study was performed with approval and in accordance with the German National Guidelines for the care of laboratory animals. Hepatocytes were isolated from adult, male Lewis rats (Charles River, Sulzfeld, Germany) weighing 200-300 g as described before (Pollok, 2003; Torok et al., 2001a,b, 2006), to obtain single liver cell suspension. After filtering through a nylon mesh with $100 \mu \mathrm{m}$ pore size, hepatocytes were separated by centrifugation at $50 \mathrm{~g}$ for 5 min and washed with Williams Medium E without glutamine (Invitrogen). Cell number and viability were determined by trypan blue test.

\section{Cell Seeding}

Our preliminary investigations with a static seeding method, where defined numbers of cells were pipetted on the scaffold, resulted in a low seeding efficiency. Therefore, a centrifugal force seeding method (Dvir-Ginzberg et al., 2003; Yang et al., 2001) was used to achieve higher cell density and to 
facilitate cell-to-cell interactions. Hepatocytes were suspended in a defined volume of culture medium to obtain cell suspension. After placing the scaffolds in a falcon tube (BD Bioscience), they were centrifuged twice at $40 \mathrm{~g}$ for $5 \mathrm{~min}$ to entrap the cells inside the pores of the scaffold. After resuspension, the scaffolds containing hepatocytes were carefully transferred to a sterile 96-well receiver tray (Millipore, Carrigtwohill, Ireland), where $350 \mu \mathrm{L}$ culture medium was added.

Hepatocyte monolayer culture in 96-well plates was used as control. The $2 \mathrm{D}$ dishes coated with Nunclon ${ }^{\mathrm{TM}} \Delta$ Surface (Thermo Fisher Scientific, Roskilde, DK) were seeded with 200,000 hepatocytes to enable attachment of as much as possible cells per well with the purpose of a maximum loading efficiency. Two hundred microliters of medium per well was added.

\section{Culture Conditions}

Hepatocytes on nanofibrous scaffolds were cultured in Williams' Medium E (Invitrogen) supplemented with the following ingredients: $200 \mathrm{mM}$ low endotoxin L-alanyl-Lglutamine (Biochrom, Berlin, Germany), 1 M HEPES buffer (Biochrom), $100 \mathrm{mM}$ sodium pyruvate (Invitrogen), $4 \mu \mathrm{g} /$ $\mathrm{mL}$ of insulin (Invitrogen), $5 \mathrm{nM}$ dexamethasone (SigmaAldrich, St. Louis, MO), $10 \mathrm{ng} / \mathrm{mL}$ epidermal growth factor (Invitrogen), $10 \mathrm{ng} / \mathrm{mL}$ recombinant human thrombopoietin (Cell Systems, St. Katharinen, Germany), $10 \mathrm{ng} / \mathrm{mL}$ recombinant human hepatocyte growth factor (Bachem, King of Prussia, PA), 10\% heat-inactivated fetal bovine serum (Invitrogen), and 1\% penicillin/streptomycin (Invitrogen). To induce cytochrome (CYP) isoenzymes, medium was supplemented from day 3 of cell culture with $2 \%$ DMSO, $2 \mu \mathrm{M} 3-$ methylcholanthrene, and $10 \mu \mathrm{M}$ dexamethasone (Hewitt et al., 2007) (Sigma-Aldrich). The scaffolds were incubated under static conditions in a humidified atmosphere of $5 \% \mathrm{CO}_{2}$ and $95 \%$ air at $37^{\circ} \mathrm{C}$ during a culture period of 7 days. Culture medium was changed every $24 \mathrm{~h}$. The $2 \mathrm{D}$ dishes were cultured under the same conditions as the scaffolds.

\section{Histological Studies and Fluorescence Microscope Analysis}

At specified time points of culture, cell polymer specimens were harvested and embedded in Tissue-Tek (Sakura, Staufen, Germany) and cut into $18 \mu \mathrm{m}$ thick sections. After fixation in acetone (Geyer), the histological sections of the 3D constructs were stained with hematoxylin and eosin (HE) for evaluation of cell viability and with periodic acid Schiff (PAS) for estimation of glycogen-storage capacity.

In addition to light microscopy analysis, spheroid formation and hepatocyte morphology were evaluated using Cctokeratin18-FITC (Dako, Glostrup, Denmark) (1:25, 45 min incubation) for liver-cell-specific cytoskeleton (green) and Hoechst 33258 (Invitrogen) as counterstaining for viable cell nuclei (blue) (1:20,000, 1-min incubation).
The activity of liver-specific factors was analyzed by immunofluorescent staining. Goat polyclonal antibodies against hepatocyte nuclear factor 4 (HNF-4) and Connexin 32 were purchased from Santa Cruz Biotechnology (Santa Cruz, CA). HNF-4 is one of the major nuclear hepatocyte transcription factors (Li et al., 2000; Schrem et al., 2002) and was used for estimation of cell differentiation (1:200, 1-h incubation). The gap junction protein Connexin 32 was stained for demonstration of intercellular communication (Piechocki et al., 2000) (1:100, 1-h incubation). Zonula occludens protein 1 (ZO-1) is a marker for tight junctions, which in the liver indicates bile canaliculi formation. Rabbit polyclonal antibody against ZO-1 was purchased from Invitrogen (1:20, 1-h incubation). The goat-anti-rabbit and donkey-anti-goat secondary antibodies were Alexa Fluor 555-conjugated and manufactured by Invitrogen (1:400, 45min incubation). After staining, the sections were visualized by fluorescence microscopy.

\section{DNA Purification}

Cell loading efficiency and extent of hepatocyte leakage from scaffolds were quantified via DNA content measurement after creating a standard curve via DNA quantification for a range of known cell numbers of five cell series. At different time points, the scaffolds were removed from the 96-well plate into an DNAse-free Eppi and DNA was purified using the QIAamp DNA Mini Kit (Qiagen, Germantown, MD) according to the manufacturer's guideline. "Tissue protocol" was applied where scaffolds were incubated in proteinase $\mathrm{K}$ overnight in a shaking water bath at $56^{\circ} \mathrm{C}$. In case of $2 \mathrm{D}$ culture the number of attached cells were determined by isolation of DNA content from the attached cells after their removal from the 96-well dish using 0.5\% trypsin-EDTA following 5-min incubation. For DNA measurements the absorbance at $260 \mathrm{~nm}$ was monitored using Infinite M200 microplate reader with Nano Quant Plate ${ }^{\mathrm{TM}}$ (Tecan, Crailsheim, Germany).

\section{Measurement of Lactate Dehydrogenase (LDH) Release and Albumin Secretion}

Biochemical assays were established for measuring cell viability in $3 \mathrm{D}$ cell culture using nanofibrous scaffolds. In this regard 24-h culture supernatants were collected every other day of culture to determine cellular $\mathrm{LDH}$ release and albumin secretion within $24 \mathrm{~h}$. Both assays were performed in 96-well plates at room temperature. LDH activity released from damaged cells was determined in duplicates using a Cytotoxicity Detection Kit ${ }^{\text {Plus }}$ based on colorimetric measurement (Roche, Basel, Switzerland). Incubation time in dark environment was precisely complied with $10 \mathrm{~min}$. LDH standard curve was created via LDH solution from hog muscle with specific activity of $550 \mathrm{U} / \mathrm{mg}$ (Roche).

Rat albumin concentration was measured by enzymelinked immunosorbent assay (ELISA) using a rat albumin quantification kit (ICL, Newberg, OR). Albumin secretion 
was measured in duplicates and concentration was calculated according to a standard curve constructed from the kit containing albumin standard. For both tests, medium samples had been diluted until the results fall in standard curve.

\section{RNA Isolation and Reverse Transcription PCR (rt-PCR)}

After harvesting on days 1 and 7 of cell culture the seeded scaffolds were incorporated into $350 \mu \mathrm{L}$ RLT buffer (Qiagen) and 3.5 $\mu \mathrm{L} \beta$-mercaptoethanol (Sigma-Aldrich). After homogenization of the 3D constructs in a QIAshredder spin column (Qiagen), the total RNA was extracted from the lysate using RNeasy Mini Kit (Qiagen). RNA content and purity were determined by absorbance measurement at 260 and 260/280 nm, respectively, using Infinite M200 microplate reader with Nano Quant Plate ${ }^{\mathrm{TM}}$ (Tecan). For the first-strand cDNA synthesis as well as pre-amplification of cDNA, $\mathrm{RT}^{2}$ Nano PreAMP cDNA Synthesis Kit from SABiosciences (Foster City, CA) was used according to the manufacturer's guideline. After elimination of genomic DNA, first-strand cDNA synthesis reaction was performed under the following conditions: $42^{\circ} \mathrm{C}$ for $30 \mathrm{~min}, 95^{\circ} \mathrm{C}$ for $5 \mathrm{~min}$, and cooling to $4^{\circ} \mathrm{C}$ until the next step. Nano PreAMP PCR cocktail was prepared as described in the manufacturer's protocol using individual $\mathrm{RT}^{2} \mathrm{qPCR}$ primer assays (SABiosciences) for the selected genes described in Table I. The pre-amplification conditions were as follows: $95^{\circ} \mathrm{C}$ for $10 \mathrm{~min}$ and 12 cycles with $95^{\circ} \mathrm{C}$ for $15 \mathrm{~s}$ and $60^{\circ} \mathrm{C}$ for $2 \mathrm{~min}$.

\section{Real-Time PCR}

Real-time PCR amplification was deployed to quantify the gene expression of liver-cell-specific factors by using the $\mathrm{RT}^{2}$ SYBR Green/ROX qPCR Master Mix in combination with gene-specific validated $\mathrm{RT}^{2}$ qPCR primer assays (SABiosciences) for optimal results. The details for the target genes are listed in Table I. The expression of liver-cellspecific factors was quantified using the comparative $C_{t}$ method, which calculates the gene expression to an internal housekeeping gene. Glyceraldehyde-3-phosphate-dehydrogenase $(\mathrm{GAPDH})$ was used as the internal control due to their stable expression. All reactions were performed in duplicates and consisted of $12.5 \mu \mathrm{L} \mathrm{RT}^{2}$ SYBR Green/ROX qPCR Master Mix, $1 \mu \mathrm{L}$ gene-specific $10 \mu \mathrm{m}$ primer pair stock containing a mix of forward and reverse primers for a specific target, and $2 \mu \mathrm{L}$ pre-amplified cDNA as PCR template in a final reaction volume of $25 \mu \mathrm{L}$. The reactions were performed using the StepOnePlus Real-Time PCR System (Applied Biosystems, Foster City, CA). The cycling conditions were as follows: $95^{\circ} \mathrm{C}$ for $10 \mathrm{~min}$ to support the initial activation of HotStart DNA polymerase followed by 45 cycles with $95^{\circ} \mathrm{C}$ for $15 \mathrm{~s}$ and $60^{\circ} \mathrm{C}$ for $1 \mathrm{~min}$. Melting curve analysis was performed routinely to verify the specificity of real-time PCR products.

\section{Statistical Analysis}

Unpaired Student's $t$-test was used to compare the results from 3D culture using nanofibrous scaffolds with monolayer culture. $P<0.05$ was accepted as significant, whereas $P<0.01$ was received as highly significant.

\section{Results}

\section{Hepatocyte Immobilization in Polymer Pores}

A mean cell viability of $86.8 \pm 2.0 \%$ was detected by trypan blue test immediately following hepatocyte isolation from adult male Lewis rats. After cell seeding by the centrifugation technique scaffolds looked as demonstrated in Figure $1 \mathrm{~A}$ and $\mathrm{B}$.

Hepatocyte distribution inside the nanofibrous scaffold was noticed using transmission light microscopy during the entire culture period. Numerous hepatocytes were found to be immobilized in polymer pores even after 7 days of culture (Fig. 1C). Furthermore, compared to the time point directly after cell seeding, transmission light microscopy revealed no detectable morphological changes during $3 \mathrm{D}$ culture in contrast to monolayer culture, where a considerable flattening and a changed morphology were detected (Fig. 1D).

Table I. Primers applied for pre-amplification of cDNA and accomplishment of real-time PCR.

\begin{tabular}{|c|c|c|c|}
\hline Gene name & $\begin{array}{l}\text { Gene } \\
\text { symbol }\end{array}$ & $\begin{array}{l}\text { Amplicon } \\
\text { length (bp) }\end{array}$ & $\begin{array}{l}\text { Ref. } \\
\text { seq. }\end{array}$ \\
\hline $\begin{array}{l}\text { Cytochrome P450, family } 2 \text {, } \\
\text { subfamily b, polypeptide } 2\end{array}$ & Cyp2b2 & 158 & XM_001070953.1 \\
\hline $\begin{array}{l}\text { Cytochrome P450, family } 3 \text {, } \\
\text { subfamily a, polypeptide } 23 / \text { polypeptide } 1\end{array}$ & Сур3a23/3a1 & 123 & NM_013105.2 \\
\hline $\begin{array}{l}\text { Cytochrome P450, family } 1 \text {, } \\
\text { subfamily a, polypeptide } 1\end{array}$ & Cyplal & 196 & NM_012540.2 \\
\hline $\begin{array}{l}\text { Cytochrome P450, family } 1 \text {, } \\
\text { subfamily a, polypeptide } 2\end{array}$ & Cypla2 & 111 & NM_012541.3 \\
\hline Albumin & Alb & 128 & NM_134326.2 \\
\hline Glyceraldehyde-3-phosphate dehydrogenase & Gapdh & 172 & NM_017008.3 \\
\hline
\end{tabular}




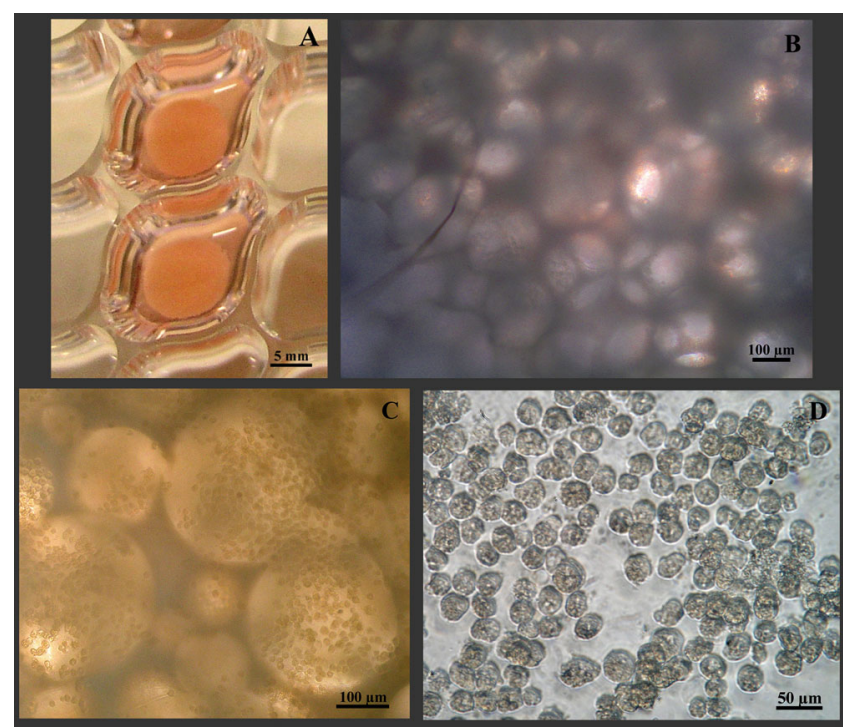

Figure 1. Nanofibrous scaffolds directly after seeding with primary rat hepatocytes by centrifugation technique (A/B) and after 7 days in $3 \mathrm{D}$ culture (C) compared to $2 \mathrm{D}$ culture (D). A: Scaffolds in a special 96-well receiver tray with $350 \mu \mathrm{L}$ modified Williams' medium E. B: Transmission light microscopy image. The high density of the nanofibrous scaffold reduces the transmitted light and the image quality. C: Transmis sion light microscopy after 7 days in 3D culture using nanofibrous scaffolds. The pores are randomly filled with cells; no morphological changes were noticed. D: Transmission light microscopy image of the $2 \mathrm{D}$ cultured cells in a 96-well culture dish after 7 days. The cells are flattened.

\section{Histological Studies and Fluorescence Microscope Analysis}

Cell viability was evaluated by HE, staining the cytoplasm pink and nucleoli blue. Primary rat hepatocytes cultured on nanofibrous scaffolds revealed intact cell micro-architecture with high viability, central nucleoli, and eosinophilic cytoplasm during the entire culture period of 7 days
(Fig. 2A). Glycogen-storage capacity is a hepatocyte-specific function. Positive PAS reaction revealed well-preserved glycogen storage on day 7 of 3D hepatocyte cultures (Fig. 2B).

3D cultured rat hepatocytes using nanofibrous scaffolds remained round with an intact cytoskeleton and formed small cell aggregates within the pores of the nanofibrous scaffold, a condition precedent for re-establishing cell-tocell communication and maintenance of hepatocyte-specific function (Fig. 3A).

HNF-4 is one of the major liver-enriched nuclear hepatocyte transcription factors in normal liver tissue (Dean et al., 2010; Li et al., 2000; Lindros et al., 1997; Schrem et al., 2002). After cell isolation, all hepatocytes initially remain HNF-4 positive. During 2D monolayer cell culture HNF-4 expression is downregulated and the staining subsequently turns negative. Within our 3D culture system, using collagen-coated nanofibrous scaffolds, hepatocytes remain HNF-4 positive during the entire cell culture period of 7 days (Fig. 3B).

ZO-1 is a marker for tight junctions that indicate bile canaliculi formation between two adjacent hepatocytes in the liver. Interestingly, this marker also displays positive as an indication for re-formation of bile canaliculi and reestablishment of the apical and basolateral membrane and, therefore, bipolar configuration of the cultured hepatocytes (Abu-Absi et al., 2002; Fanning and Anderson, 2009). We detected ZO-1-positive hepatocytes during the entire culture period in $3 \mathrm{D}$ culture in those hepatocytes, which are organized in clusters (Fig. 3C), where ZO-1 is obviously visible between adjacent cells as two parallel stripes defining bile canaliculi (Abu-Absi et al., 2002; Fanning and Anderson, 2009).

The gap junction protein Connexin 32 is a marker for highly differentiated hepatocyte interaction, enabling cellto-cell communication (Piechocki et al., 2000). Aggregated hepatocytes in our 3D culture system re-established positive signaling for this marker within 7 days of culture (Fig. 3D).

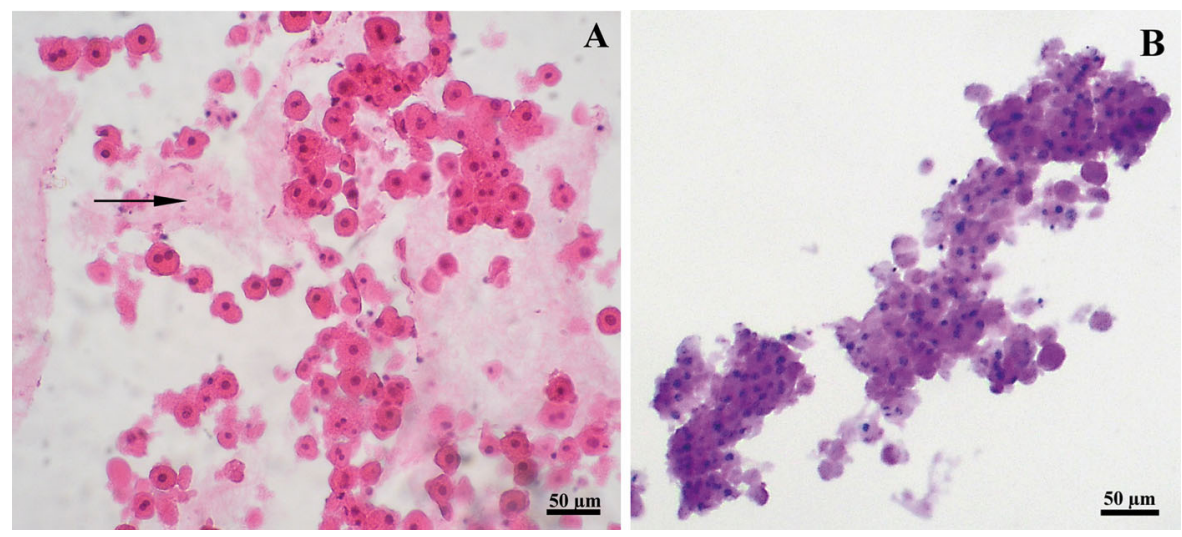

Figure 2. Histological analysis after 7 days in 3D culture using nanofibrous scaffolds. A: HE staining revealed high cell viability and close cell-to-cell contacts; the polymer visible in the background is marked with an arrow. B: PAS staining shows well-preserved glycogen storage. 

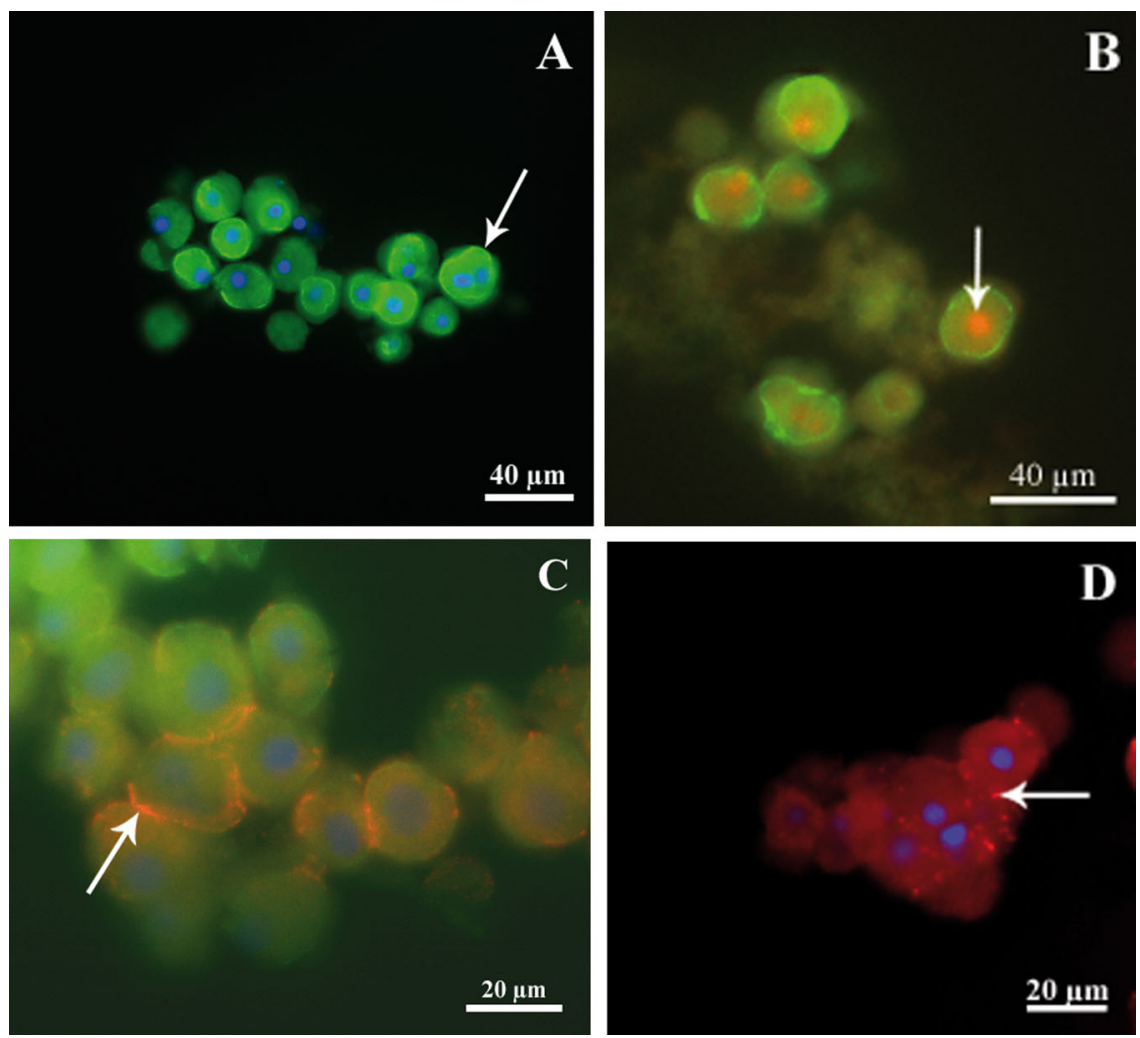

Figure 3. Immunofluorescent staining of hepatocyte-specific factors (white arrows) after 7 days in 3D culture. A: CK18 (green)/DAPI (blue) double staining reveals intact nucleus and cytoskeleton. B: Double staining of liver-cell-specific transcription factor HNF-4 (red) and cytokeratin 18 (green). Positive nuclear staining of HNF-4 indicates highly preserved cell differentiation. C: ZO-1 (red)/Hoechst 33258 (blue)/CK18 (green) staining indicating neo formation of bile canaliculi and bipolar configuration of the aggregated hepatocytes within the nanofibrous scaffold. D: Immunofluorescent staining of the gap junction protein Connexin 32 (red). Hoechst 33258 stained viable nuclei blue. Cell-to-cell contacts could be re-established between flanking cells.

Contrary to the results in 3D culture, immunohistological staining in the $2 \mathrm{D}$ cultured rat hepatocytes was negative for HNF-4, ZO-1, and Connexin 32 as expected (pictures not shown).

\section{Cell Loading Efficiency}

One of our aims was to achieve maximal cell density in our respective culture systems. A centrifugal force seeding method was used for all 3D experiments presented at this juncture. For comparison, monolayer culture with 200,000 cells was performed in an adhesive 96-well culture dish. Figure 4B summarizes the results of DNA content measurement, comparing $2 \mathrm{D}$ and $3 \mathrm{D}$ cultures. Centrifugal force seeding achieved a high loading efficiency with $57,000 \pm 39,000$ cells per scaffold. In 2D culture 34,000 $\pm 8,000$ cells attached per well on day 1 . On day 7 , an average of $20,000 \pm 12,000$ cells was detected per scaffold via DNA content measurement, whereas $24,000 \pm 14,000$ cells remained attached in monolayer culture (values not significant).

\section{Albumin Secretion and LDH Release}

Albumin content per well was monitored via ELISA detection system. Figure 5 depicts the kinetic of 24-h albumin secretion during the 7-day culture period in $3 \mathrm{D}$ or $2 \mathrm{D}$ culture, respectively. Albumin in medium samples was detectable during the entire culture period, but the levels evenly decreased from $1,815 \pm 520 \mathrm{ng} /$ well on day 1 in $3 \mathrm{D}$ culture $(336 \pm 118 \mathrm{ng} /$ well in $2 \mathrm{D}$ culture) to $138 \pm 94 \mathrm{ng} /$ well on day $7(15 \pm 13 \mathrm{ng} /$ well, respectively; Fig. 5A). The same was found, taking into consideration the cell numbers per well: albumin secretion per $1 \times 10^{6}$ cells was lower in the cells cultured in $2 \mathrm{D}$ dishes than those secreted by the cells in $3 \mathrm{D}$ culture at the beginning $(12,077 \pm 8,730 \mathrm{ng}$ in $2 \mathrm{D}$ vs. $47,213 \pm 33,203 \mathrm{ng}$ in $3 \mathrm{D}$ culture $)$ as well as at the end of cell culture $(2,128 \pm 3,304 \mathrm{ng}$ vs. $10,668 \pm 8,095 \mathrm{ng} ;$ Fig. 5B). The differences were highly significant $(P<0.01)$.

LDH activity was determined by a method based on colorimetric measurement. Release of $\mathrm{LDH}$ decreased permanently with time from high levels of $21,283 \pm 8,843 \mu \mathrm{g} /$ well on day 1 to low levels of $1,230 \pm 365 \mu \mathrm{g} /$ well on day 5 and was nearly undetectable on day 7 of culture, thus indicating 
A DNA standard curve created from 5 cell series

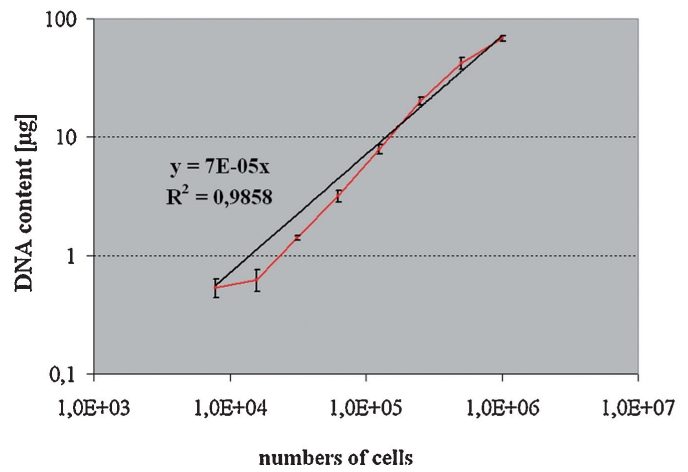

B DNA-content per polvmer/well

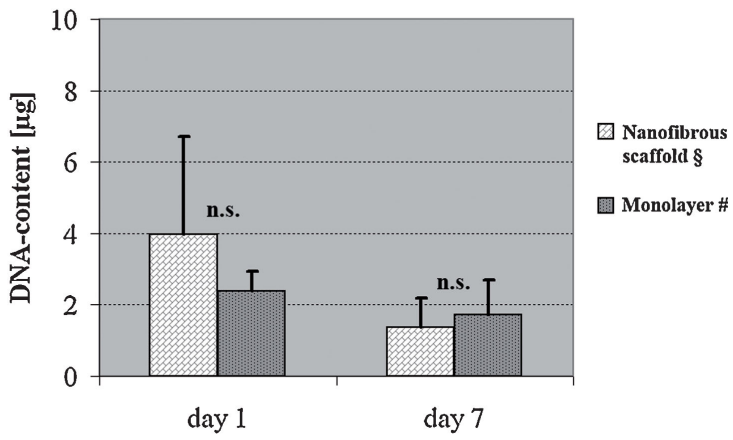

Figure 4. DNA content measurement. A: Standard curve for known numbers of cells created from five series of rat hepatocytes. The values are presented as mean \pm SD. B: DNA content in nanofibrous scaffolds after seeding with centrifugation technique (tiled columns) enables loading efficiency of nearly 57,000 cells per well in a 96 -well plate. The data are expressed as mean + SD. The values comparing nanofibrous 3D culture and monolayer culture are not significant (n.s.). The DNA content from 16 scaffolds per time point was measured (\$). The hepatocytes are derived from three isolations. For monolayer culture, 12 wells were measured for each time point (\#), with the hepatocytes deriving from two isolations.

that the cells remaining on the nanofibrous scaffold display superior viability (Fig. 6). The differences between the two groups were highly significant $(P<0.01)$. culture (Fig. 7). Decreased CYP1A1 expression was detected on day 7 in our $3 \mathrm{D}$ culture system in comparison with monolayer culture (data not significant).

\section{Real-Time PCR}

Gene expression levels of liver-derived factors have been evaluated by quantitative real-time PCR using GAPDH as the internal control. In comparison with day 1 an increased albumin mRNA expression on day 7 of 3D culture was determined, whereas albumin expression in 2D culture slightly decreased (Fig. 7). After treatment with 3-methylcholanthrene and dexamethasone, induction of CYP isoenzyme expression was more successful in 3D culture using nanofibrous scaffolds, where the compounds caused more stable (CYP3A1, CYP1A2) or even increasing (CYP2B2) enzyme expression than in 2D

\section{Discussion}

Primary rat hepatocyte culture is currently the most sensitive method for toxicity studies, and rat liver-derived model systems are commonly used for drug development and investigation of hepatotoxicity. However, the utility of rat hepatocytes for toxicological studies has been limited by morphologic and functional changes of cells cultured in 2D systems (LeCluyse et al., 2005). In those conventional cultures, a strong perturbation of cell morphology with fibroblast-like protrusions, increasing nuclear volume and granulated cytoplasm

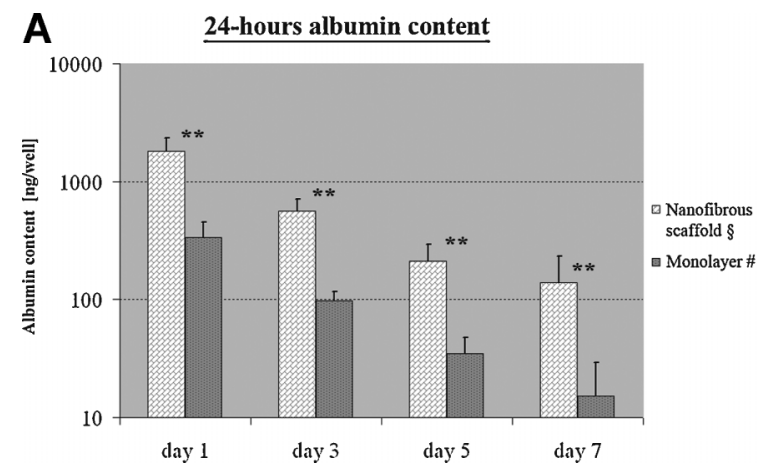

B 24-hours albumin content per $1 \times 10^{6}$ cells

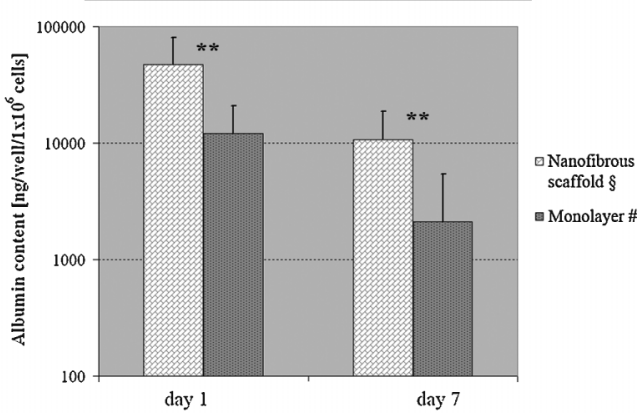

Figure 5. Twenty-four-hour albumin secretion of hepatocytes cultured on nanofibrous scaffolds (tiled columns) in contrast to monolayer culture (dotted columns). The values are represented as mean $+\mathrm{SD}$. All the differences between the two groups are highly significant at the $P<0.01$ level $\left({ }^{* *}\right)$. A: Decreasing secretion rates per well during different time points were detected in both culture systems. B: Taking into consideration the cell numbers per well, albumin secretion per $1 \times 10^{6}$ cells was higher in the $3 \mathrm{D}$ culture at the beginning as well as at the end of cell culture. The supernatant from 16 scaffolds per time point was measured for albumin content (\$). The hepatocytes are derived from three isolations. For monolayer culture 12 wells were measured for each time point (\#), with the hepatocytes deriving from two isolations. 


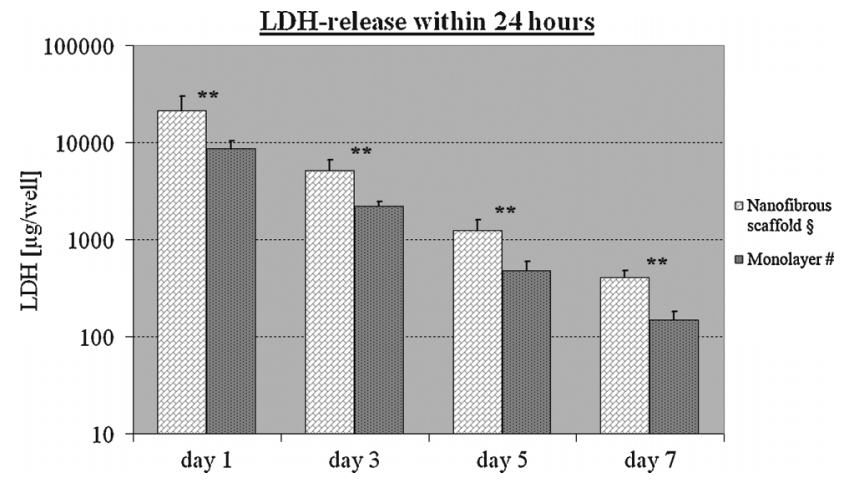

Figure 6. Total LDH content measurement. Levels decreased permanently with culture duration, indicating the presence of cells with excellent viability on culture day 7. The differences between the two groups are highly significant at the $P<0.01$ level ${ }^{(* *)}$. On days 1 and 7 the supernatant from 16 scaffolds, on days 3 and 5 from 12 scaffolds, was measured for LDH release (\$). The hepatocytes are derived from three isolations. For monolayer culture, 12 wells were measured for each time point (\#), with the hepatocytes deriving from two isolations.

have been found already after $72 \mathrm{~h}$ in $2 \mathrm{D}$ culture (Hewitt et al., 2007). This experience is in accordance with our existing observations, where primary rat hepatocytes cultured in 2D dishes showed several changes in cytoarchitecture. Contrary to monolayer culture, rat hepatocytes cultured on nanofibrous scaffolds exhibited no obvious morphological changes during the 7-day culture period as demonstrated by transmission light microscopy and HE staining. Hewitt et al. (2007) propose evaluation of more parameters than metabolization, for example, cell morphology, glycogen-storage capacity, or expression and localization of individual proteins to characterize the type of liver toxicity and hepatocyte injury. This is in agreement with our experiences in hepatocyte culture, where morphological stability is essential for perpetuation of cell-specific hepatocyte function like glycogen detention. Hepatocytes cultured on nanofibrous scaffolds showed well-preserved glycogen storage until the last day of culture, as verified by positive PAS reaction of nearly all hepatocytes after 7 days in 3D culture.

Pharmaceutical industry requires effective high-throughput assays. Furthermore, large initially cell numbers are required to restore normal cell communication and therefore cell function. Otherwise the growth area in one well of a 96-well microplate is very restricted and permits only low cell numbers. Altogether this means a big challenge for scientists to meet these claims, especially in the case of cells with low in vitro proliferative ability like hepatocytes. To create a functional $3 \mathrm{D}$ hepatocyte culture was the purpose of this study, to achieve a strong correlation between the in vivo and in vitro situation of cells. We used centrifugal force for seeding and attaining an average cell number of nearly $57,000 \pm 39,000$ cells per well in a 96-well plate and, this is the most important fact that these cells were of excellent viability as demonstrated by HE staining. However, to provide that many cells in a 96-well plate with nutritive substances is a limiting factor in cell culture. This technical problem was overcome by the application of a special 96well culture dish with lengthwise oval-shaped wells (Millipore), where $350 \mu \mathrm{L}$ culture medium fit well into, initially suggested as a receiver tray. This tray allows easy removal of the scaffolds from the wells for further analysis, without causing damage to the scaffold, and cells within. Secondly, the oval shape configuration increases the volume of the well and thereby the surface to volume ratio of the culture medium to permit better gas exchange during cell culture (Mikos et al., 1993; Provin et al., 2008).

Total LDH content per well indirectly reflects the number of dead cells, due to an immediate enzyme release from the cytosol of damaged cells. Therefore, we used the LDH assay as a useful biochemical index to observe histological changes correlating with cellular membrane damage and hepatocyte injury. On the other hand, a screening of toxicological effects caused by the scaffolds and their degradation products on
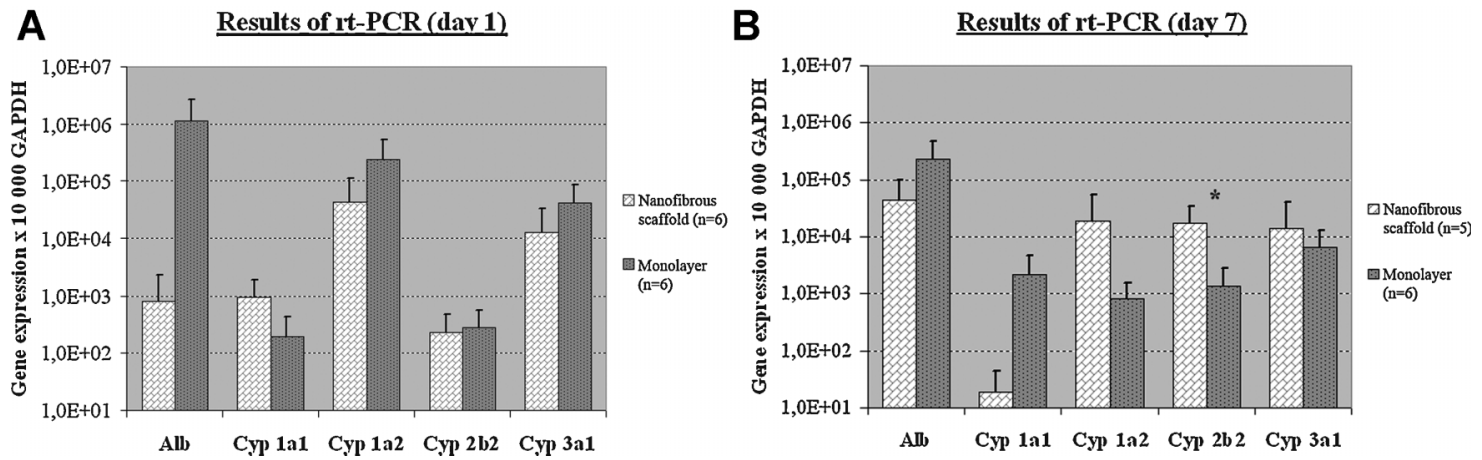

Figure 7. Gene expression pattern of hepatocytes cultured on nanofibrous scaffolds in contrast to monolayer after 1 (A) and 7 (B) days of culture expressed as mean + SD. Total RNA was extracted from hepatocytes, reverse transcribed, pre-amplified, and amplified by quantitative real-time PCR. The differences for CYP2B2 on day 7 are significant at the $P<0.05$ level (*). 
primary rat hepatocytes used in our $3 \mathrm{D}$ culture system is necessary. The high levels of $\mathrm{LDH}$ leakage into the extracellular medium at the first culture days indicate the mobilization and therefore the loss of cells that were not incorporated into the pores of the nanofibrous scaffold. Due to the consequently decreased levels of LDH leakage in combination with a high DNA content per scaffold after 7 days in 3D culture, adverse effects of the nanofibrous biomaterial may be excluded. Albumin production and secretion are quantified in general for estimation of capability of liver in vitro models. Regarding albumin secretion per well the presented results seem to indicate a loss of function in both culture systems. However, taking the cell numbers per well into account, albumin content in relation to cell number is significantly higher in 3D culture compared to monolayer culture. Furthermore, we suppose that the high levels of albumin, measured in the culture supernatant during the first days of culture, are falsely positive with regard to albumin production, due to release of intracellular albumin from damaged or necrotic cells. The ELISA method measures albumin content rather than production and cannot distinguish between albumin release from death cells and active protein production from vital hepatocytes with active and specific cell metabolism. This assumption is supported by the observed increase in albumin mRNA expression during day 7 of 3D culture. We therefore suggest that in reality the decrease of albumin protein detected during the culture period does not reflect similar scale loss of cellular albumin production, as the data implicate.

Nanofibrous scaffolds with interconnected spherical pore networks were initially evaluated using osteoblastic progenitor cells (Ma, 2004; $\mathrm{Ma}$ and Choi, 2001). In tissue engineering, the scaffold serves as a $3 \mathrm{D}$ template supporting cell attachment, proliferation, differentiation, and neo tissue regeneration. We therefore established a series of immunohistological studies to examine cell-to-cell interactions and reorganization or neo tissue regeneration within the pores of the nanofibrous scaffold. HNF-4 plays an important role in the liver, due to its regulation of gene expression into proteins including development, differentiation, and its involvement in diverse metabolic pathways. The presence of HNF-4 protein has been correlated with the expression of the liver phenotype in vitro (Schrem et al., 2002). Positive nuclear staining of cells on nanofibrous scaffolds provides the convincing evidence of highly differentiated primary rat hepatocytes existing in scaffold pores after 7 days of culture.

Connexin 32 is a major hepatic gap junction protein enabling cell-to-cell communication (Piechocki et al., 2000). The disappearance of connexin-mediated gap junctions in primary rat hepatocytes cultured in monolayers has been described previously (Hamilton et al., 2001; Hou et al., 2001). A uniform pattern of intercellular gap junctions between adjacent hepatocytes cultured on nanofibrous scaffolds was observed after 7 days of 3D culture, strongly suggesting the ability of highly differentiated hepatocyte interaction. Close cell-to-cell contacts play a critical role in determining normal responsiveness to chemical modulators (Hamilton et al., 2001).

Hepatocytes are highly polarized cells depending on the maintenance of sinusoidal and canalicular membrane domains separated by tight junctions (LeCluyse et al., 2005). ZO-1 is known as a tight junction associated polypeptide. It is well known that freshly isolated primary rat hepatocytes loose their polarized structure directly after dissociation from tissue. Detection of ZO-1 positive hepatocytes cultured on nanofibrous scaffolds indicates re-formation of bile canaliculi and bipolar configuration. The preservation of a polarized cell and membrane architecture is essential for biliary excretion and xenobiotic elimination (Abu-Absi et al., 2002; LeCluyse et al., 2005).

Expression of CYP isoenzymes is generally downregulated in conventional cultures from the third culture day as a consequence of dedifferentiation (Donato and Castell, 2003; Hewitt et al., 2007; Tuschl and Mueller, 2006). The results of real-time PCR after 7 days of 3D culture using nanofibrous scaffolds evidence the presence of highly differentiated and functional hepatocytes with the capability of metabolizing toxic substances. However, the loss of CYP1A1 mRNA expression has to be discussed. On one hand, CYP mRNA expression evaluated by real-time PCR may not always reflect the levels of the catalytically active enzyme present in cells (Donato and Castell, 2003). On the other hand, there is a strong effect of culture conditions on CYP expression and the number of well-analyzed CYP inhibitors is low. Tuschl and Mueller (2006) found strongly increased CYP1A1 levels in serum-free and considerably decreased CYP levels in serum-containing cultures. Possibly the usage of serum-free culture medium may also increase CYP1A1 expression in our 3D culture system. Nevertheless, xenobiotics are consequently metabolized by several CYP isoenzymes (Donato and Castell, 2003).

\section{Conclusion}

The presented data from our study suggest that nanofibrous scaffolds with interconnected spherical macropores provide a good in vitro microenvironment for viability and neo tissue formation of primary rat hepatocytes, preserving specific enzymatic function and morphology. Our results are in agreement with previous studies (Hamilton et al., 2001) showing that the matrix configuration has a pronounced effect on maintenance of an in vivo like hepatocyte morphology and function even in short time culture.

The authors wish to acknowledge the National Institutes of Health (NIH) for financial support (NIGMS GM075840: P.X.M.). Furthermore, the authors thank Ms. Roshni Goswami for technical assistance.

\section{References}

Abu-Absi SF, Friend JR, Hansen LK, Hu WS. 2002. Structural polarity and functional bile canaliculi in rat hepatocyte spheroids. Exp Cell Res 274(1):56-67. 
Aly HA, Domenech O. 2009. Aroclor 1254 induced cytotoxicity and mitochondrial dysfunction in isolated rat hepatocytes. Toxicology 262(3):175-183.

Chen VJ, Ma PX. 2004. Nano-fibrous poly(L-lactic acid) scaffolds with interconnected spherical macropores. Biomaterials 25(11):2065-2073.

Chen VJ, Smith LA, Ma PX. 2006. Bone regeneration on computer-designed nano-fibrous scaffolds. Biomaterials 27(21):3973-3979.

Dean S, Tang JI, Seckl JR, Nyirenda MJ. 2010. Developmental and tissuespecific regulation of hepatocyte nuclear factor 4-alpha (HNF4-alpha) isoforms in rodents. Gene Expr 14(6):337-344.

Donato MT, Castell JV. 2003. Strategies and molecular probes to investigate the role of cytochrome P450 in drug metabolism: Focus on in vitro studies. Clin Pharmacokinet 42(2):153-178.

Dvir-Ginzberg M, Gamlieli-Bonshtein I, Agbaria R, Cohen S. 2003. Liver tissue engineering within alginate scaffolds: Effects of cell-seeding density on hepatocyte viability, morphology, and function. Tissue Eng 9(4):757-766.

Ellis JK, Chan PH, Doktorova T, Athersuch TJ, Cavill R, Vanhaecke T, Rogiers V, Vinken M, Nicholson JK, Ebbels T, Keun HC. 2010. Effect of the histone deacetylase inhibitor trichostatin a on the metabolome of cultured primary hepatocytes. J Proteome Res 9(1):413-419.

Fanning AS, Anderson JM. 2009. Zonula occludens-1 and -2 are cytosolic scaffolds that regulate the assembly of cellular junctions. Ann N Y Acad Sci 1165:113-120.

Gebhardt R, Hengstler JG, Muller D, Glockner R, Buenning P, Laube B, Schmelzer E, Ullrich M, Utesch D, Hewitt N, et al. 2003. New hepatocyte in vitro systems for drug metabolism: Metabolic capacity and recommendations for application in basic research and drug development, standard operation procedures. Drug Metab Rev 35(2-3):145-213.

Hamilton GA, Jolley SL, Gilbert D, Coon DJ, Barros S, LeCluyse EL. 2001. Regulation of cell morphology and cytochrome P450 expression in human hepatocytes by extracellular matrix and cell-cell interactions. Cell Tissue Res 306(1):85-99.

Hertzberg RP, Pope AJ. 2000. High-throughput screening: New technology for the 21st century. Curr Opin Chem Biol 4(4):445.

Hewitt NJ, Lechon MJ, Houston JB, Hallifax D, Brown HS, Maurel P, Kenna JG, Gustavsson L, Lohmann C, Skonberg C, et al. 2007. Primary hepatocytes: Current understanding of the regulation of metabolic enzymes and transporter proteins, and pharmaceutical practice for the use of hepatocytes in metabolism, enzyme induction, transporter, clearance, and hepatotoxicity studies. Drug Metab Rev 39(1):159-234.

Hou DX, Arimura M, Fukuda M, Oka T, Fujii M. 2001. Expression of cell adhesion molecule and albumin genes in primary culture of rat hepatocytes. Cell Biol Int 25(3):239-244.

LeCluyse EL, Alexandre E, Hamilton GA, Viollon-Abadie C, Coon DJ, Jolley S, Richert L. 2005. Isolation and culture of primary human hepatocytes. Methods Mol Biol 290:207-229.

Lee J, Cuddihy MJ, Cater GM, Kotov NA. 2009. Engineering liver tissue spheroids with inverted colloidal crystal scaffolds. Biomaterials 30(27):4687-4694.

Li J, Ning G, Duncan SA. 2000. Mammalian hepatocyte differentiation requires the transcription factor HNF-4alpha. Genes Dev 14(4):464474.

Li Y, Qi XM, Xue X, Wu XF, Wu YF, Chen M, Xing GZ, Luan Y, Ren J. 2009. The relationship between diphenylamine structure and NSAIDsinduced hepatocytes injury. Toxicol Lett 186(2):111-114.

Lindros KO, Oinonen T, Issakainen J, Nagy P, Thorgeirsson SS. 1997. Zonal distribution of transcripts of four hepatic transcription factors in the mature rat liver. Cell Biol Toxicol 13(4-5):257-262.

Liu X, Won Y, Ma PX. 2006. Porogen-induced surface modification of nano-fibrous poly(L-lactic acid) scaffolds for tissue engineering. Biomaterials 27(21):3980-3987.

Ma PX. 2003. Tissue engineering. Hoboken, NJ: John Wiley and Sons, Inc. Ma PX. 2004. Scaffolds for tissue fabrication. Mater Today 7(5):30-40.

Ma PX. 2008. Biomimetic materials for tissue engineering. Adv Drug Deliv Rev 60(2):184.
Ma PX, Choi JW. 2001. Biodegradable polymer scaffolds with well-defined interconnected spherical pore network. Tissue Eng 7(1):23-33.

Ma PX, Zhang R. 1999. Synthetic nano-scale fibrous extracellular matrix. J Biomed Mater Res 46(1):60-72.

Mikos AG, Bao Y, Cima LG, Ingber DE, Vacanti JP, Langer R. 1993. Preparation of poly(glycolic acid) bonded fiber structures for cell attachment and transplantation. J Biomed Mater Res 27(2):183-189.

Piechocki MP, Toti RM, Fernstrom MJ, Burk RD, Ruch RJ. 2000. Liver cellspecific transcriptional regulation of connexin 32. Biochim Biophys Acta 1491(1-3):107-122.

Pollok JM. 2003. Engineering of liver tissue within a dynamic flow culture bioreactor for transplantation. University Medical Center HamburgEppendorf.

Pollok JM, Kluth D, Cusick RA, Lee H, Utsunomiya H, Ma PX, Langer R, Broelsch CE, Vacanti JP. 1998. Formation of spheroidal aggregates of hepatocytes on biodegradable polymers under continuous-flow bioreactor conditions. Eur J Pediatr Surg 8(4):195-199.

Provin C, Takano K, Sakai Y, Fujii T, Shirakashi R. 2008. A method for the design of 3D scaffolds for high-density cell attachment and determination of optimum perfusion culture conditions. J Biomech 41(7):14361449.

Santos DM, Santos MM, Viana RJ, Castro RE, Moreira R, Rodrigues CM. 2009. Naphtho[2,3-d]isoxazole-4,9-dione-3-carboxylates: Potent, noncytotoxic, antiapoptotic agents. Chem Biol Interact 180(2):175-182.

Schrem H, Klempnauer J, Borlak J. 2002. Liver-enriched transcription factors in liver function and development. Part I: The hepatocyte nuclear factor network and liver-specific gene expression. Pharmacol Rev 54(1):129-158.

Shitara Y, Nagamatsu Y, Wada S, Sugiyama Y, Horie T. 2009. Long-lasting inhibition of the transporter-mediated hepatic uptake of sulfobromophthalein by cyclosporin a in rats. Drug Metab Dispos 37(6):1172-1178.

Smith LA, Ma PX. 2004. Nano-fibrous scaffolds for tissue engineering. Colloids Surf B Biointerfaces 39(3):125-131.

Torok E, Pollok JM, Ma PX, Kaufmann PM, Dandri M, Petersen J, Burda MR, Kluth D, Perner F, Rogiers X. 2001a. Optimization of hepatocyte spheroid formation for hepatic tissue engineering on three-dimensional biodegradable polymer within a flow bioreactor prior to implantation. Cells Tissues Organs 169(1):34-41.

Torok E, Pollok JM, Ma PX, Vogel C, Dandri M, Petersen J, Burda MR, Kaufmann PM, Kluth D, Rogiers X. 2001b. Hepatic tissue engineering on 3-dimensional biodegradable polymers within a pulsatile flow bioreactor. Dig Surg 18(3):196-203.

Torok E, Pollok JM, Ma PX, Kaufmann PM, Dandri M, Petersen J, Burda MR, Kluth D, Perner F, Rogiers X. 2002. Pre-implantation optimization of culture conditions for hepatocytes in a flow bioreactor for hepatic tissue engineering on 3-dimensional biodegradable polymers. Zentralbl Kinderchir 11:188-194.

Torok E, Vogel C, Lutgehetmann M, Ma PX, Dandri M, Petersen J, Burda MR, Siebert K, Dullmann J, Rogiers X, et al. 2006. Morphological and functional analysis of rat hepatocyte spheroids generated on poly(Llactic acid) polymer in a pulsatile flow bioreactor. Tissue Eng 12(7):1881-1890.

Tuschl G, Mueller SO. 2006. Effects of cell culture conditions on primary rat hepatocytes-Cell morphology and differential gene expression. Toxicology 218(2-3):205-215.

Wei G, Ma PX. 2006. Macroporous and nanofibrous polymer scaffolds and polymer/bone-like apatite composite scaffolds generated by sugar spheres. J Biomed Mater Res A 78(2):306-315.

Woo KM, Chen VJ, Ma PX. 2003. Nano-fibrous scaffolding architecture selectively enhances protein adsorption contributing to cell attachment. J Biomed Mater Res A 67A (2):531-537.

Woo KM, Jun JH, Chen VJ, Seo J, Baek JH, Ryoo HM, Kim GS, Somerman MJ, Ma PX. 2007. Nano-fibrous scaffolding promotes osteoblast differentiation and biomineralization. Biomaterials 28(2):335-343.

Yang TH, Miyoshi H, Ohshima N. 2001. Novel cell immobilization method utilizing centrifugal force to achieve high-density hepatocyte culture in porous scaffold. J Biomed Mater Res 55(3):379-386. 